\title{
Percepções dos Profissionais sobre a Atuação dos Psicólogos nas Unidades Básicas de Saúde ${ }^{1}$
}

\author{
Francielle Xavier Dias \\ Universidade de São Paulo, SP, Brasil.
}

\author{
Luiz Carlos Avelino da Silva \\ Universidade Federal de Uberlândia, MG, Brasil.
}

\begin{abstract}
Resumo: Este artigo discute o papel dos psicólogos na saúde pública a partir da visão dos profissionais que atuam junto a eles nas Unidades Básicas deSaúde(UBS).Oobjetivo foi investigar a visão desses profissionais sobre o papel e a importância da atuação do psicólogo. Trata-se de uma pesquisa qualitativa em que os dados analisados são resultantes de interações entre o investigador e os participantes da pesquisa. Foram realizadas entrevistas semiestruturadas, baseadas em um roteiro, com 14 sujeitos de categorias profissionais distintas, escolhidos aleatoriamente em um universo de outros 54 sujeitos que responderam a um questionário anterior. Os resultados mostram o psicólogo como um profissional considerado importante e necessário às Unidades Básicas de Saúde. Porém, com uma identidade ambígua, devido às diferentes demandas para as quais é chamado a atender, relativas à saúde mental e à atenção primária. Nesse contexto, é esperado que o psicólogo cumpra um papel de mediador.
\end{abstract}

Palavras-chave: Atuação do Psicólogo, Serviços de Saúde, Saúde Mental, Atenção Primária à Saúde.

\section{Perceptions of Other Professionals about the Work of Psychologists in Health Basic Units}

\begin{abstract}
This article discusses the role of psychologists in public health, from the perspective of other professionals working in Basic Health Units (BHU). The aim was to investigate the vision of these professionals about the role of the psychologist and about the importance of their work. Data of this qualitative study was a result of interactions between researcher and participants. Semi structured interviews were carried out based on a script, with 14 subjects from different professional categories who were randomly chosen from a universe of 54 persons that had responded to a previous questionnaire. The results show the psychologist is considered as an important and necessary professional to Health Basic Units. However, an ambiguous identity attributed to psychologists was revealed, due to the different demands they have to face, regarding both mental health and primary healthcare. In this context, it is expected that psychologists perform the role of moderators.
\end{abstract}

Keywords: Acting of the Psychologist, Health Services, Mental Health, Primary Health Care.

\footnotetext{
${ }^{1}$ Agradecemos o apoio da Prefeitura Municipal de Uberlândia (PMU) pela abertura para a coleta de dados, realizada nas Unidades Básicas de Saúde entre 2009 e 2010, como projeto de iniciação científica, sem bolsa, vinculado ao Programa Institucional de Apoio à Iniciação Científica (PIAIC/UFU).
} 


\title{
Percepciones de Otros Profesionales sobre la Actuación de Psicólogos en Unidades de Atención Primária de Salud
}

\begin{abstract}
Resumen: En este artículo se discutió el rol de los psicólogos en la salud pública, partiendo de la visión de otros profesionales que actúan junto a ellos en las unidades básicas de salud (UBS). El objetivo fue investigar la visión de otros profesionales sobre el rol y la importancia de la actuación del psicólogo. Se trata pues de una investigación cualitativa en la que los datos analizados son resultantes de las interacciones entre el investigador y sus colaboradores. Fueron realizadas entrevistas semiestructuradas, basadas en un guion, con 14 sujetos de categorías profesionales distintas, seleccionados aleatoriamente de un universo de 54 sujetos que habían contestado a un cuestionario anterior. Los resultados muestran al psicólogo como un profesional considerado importante y necesario en las unidades básicas de salud, aunque con una identidad ambigua, debido a las distintas demandas que debe atender, relativas a la salud mental y a la atención primaria. En este contexto, se espera que el psicólogo cumpla un rol de mediador.

Palabras clave: Actuación del Psicólogo, Servicios de Sanidad, Sanidad Mental, Atención Primaria a la Sanidade.
\end{abstract}

\section{Introdução}

Desde sua regulamentação, a Psicologia vem acompanhando os diversos desdobramentos sociais, políticose econômicosqueocasionaramemmudanças na assistência em saúde no país. Esse processo tem provocado transformações na formação em Psicologia, tendo em vista a aproximação com as diretrizes e princípios do Sistema Único de Saúde (SUS) (Portes, \& Máximo, 201012).

A inserção dos psicólogos na saúde pública brasileira é resultante de um complexo processo de transformação econômica, política e ideológica no início da década de 1970. Entre os anos de 1976 a 1984, a participação de psicólogos nesse setor experimentou uma taxa de crescimento de $21,47 \%$, ficando acima da média de outras categorias profissionais. Um novo olhar sobre o conceito de saúde propiciou condições para a inserção de profissionais de outras áreas, além dos tradicionais médicos, enfermeiros e técnicos de enfermagem (Andrade, \& Simon, 2009).

Dentre os diversos fatores considerados relevantes à inserção de psicólogos nos serviços públicos de saúde, destacam-se: 1) o contexto de políticas públicas de saúde do final dos anos 1970 e, principalmente, nos 1980;2) a diminuição da busca pelos consultórios de Psicologia, devido ao empobrecimento da população; 3) a ação da própria categoria com o intuito de redefinir a função social da Psicologia na sociedade e 4) a popularização da psico- logia na sociedade através da difusão da psicanálise. Tem-se que a conjuntura desses fatores associada à crítica ao tratamento asilar, bem como ao modelo de atenção médico-privativista-assistencial, contribuíram para a entrada do psicólogo na saúde pública (Dimenstein, 1998).

Após a Reforma Sanitária, a Psicologia ampliou seu espaço de atuação na saúde pública. $\mathrm{O}$ direito ao acesso irrestrito e universal à saúde, garantido pela Constituição Brasileira de 1988, ocasionou um processo de transformação das práticas no campo psi, essencialmente, devido à inviabilidade da aplicação do modelo da clínica tradicional.

O setor da saúde, em seus três níveis de atenção, representa, atualmente, um dos maiores campos de trabalho do psicólogo. No entanto, o processo de interlocução dos psicólogos com a saúde coletiva vem sendo problemático, remetendo a uma formação que ainda não tem fornecido a preparação adequada para a atuação (Azevedo, Tatmatsu, \& Ribeiro, 2011). Desse modo, tornou-se premente a constituição de um campo de saber orientado para as especificidades das práticas de saúde com vistas à construção de uma identidade profissional mais adequada. No cotidiano, o psicólogo se vê diante de um despreparo histórico de sua categoria, uma vez que o campo da saúde não é o campo da Psicologia por excelência.

Apesar do inegável avanço da proposta das Diretrizes Curriculares Nacionais para os cursos de gradu- 
ação em Psicologia, elas não sugerem ações durante a formação que propiciem e valorizem o trabalho em equipe, fundamental para a atuação na saúde pública (Bernardes, 2006). A formação do psicólogo ainda é pautada por uma proposta clínica tradicional, com ensino centrado no modelo intervencionista em detrimento da promoção e prevenção em saúde. Desse modo, há uma demanda por mudanças que ampliem e requalifiquem a formação em Psicologia para atuação na atenção básica (Azevedo et al., 2011).

A atuação clínica tradicional representa um resquício do processo histórico de constituição da Psicologia enquanto profissão elitista, comprometida com uma apreensão biologicista da experiência humana (Nepomucemo, \& Brandão, 2011). As práticas em saúde apontam a fragilidade da formação generalista em Psicologia, pois ainda se observa uma formação fragmentada, reducionista, individualizante e com especialização precoce. Assim, a inclusão de novos conteúdos e discussões sobre a realidade profissional na saúde ainda não apagou o sonho de profissional liberal presente na origem da Psicologia como profissão no Brasil. Particularmente, em função dos baixos salários pagos aos psicólogos que atuam na saúde pública, tornando-a atrativa, na maioria das vezes, apenas para os recém-formados.

A superação do modelo essencialmente clínico de formação é um processo lento. Apesar de ações do Ministério da Educação e Ministério da Saúde, como o Programa de Educação pelo Trabalho (PET-Saúde), buscarem uma formação contextualizada e engajada, observa-se ainda profissionais recém-formados sem sintonia com as peculiaridades do trabalho em saúde pública.

\section{Psicologia e atenção básica}

O trabalho dos psicólogos na atenção básica tem sido amplamente estudado pelos pesquisadores da área de saúde pública e da Psicologia. De modo geral, os estudos destacam a importância da contextualização da prática da Psicologia na saúde pública e a necessidade da reformulação do currículo profissional, a fim de proporcionar maior preparo para o trabalho na área (Ronzani, \& Rodrigues, 2006).

A inserção do psicólogo mobilizou transformações nas práticas psicológicas e de saúde. Ainda se observam visões estereotipadas sobre os transtornos mentais, predominância da lógica manico- mial, ausência de registros e estratégias, falta de apoio qualificado às famílias e pouca integração entre saúde mental e a rede de saúde dos municípios (Souza, Menandro, Couto, Schimith, \& Lima, 2012). Esses aspectos indicam a necessidade de se investir na melhoria da articulação entre saúde mental e saúde da família.

A falta de percepção de que os conhecimentos podem ser produzidos ou ressignificados na prática coletiva representa grande dificuldade para os profissionais atuantes no SUS. Uma postura profissional orientada para o cotidiano simboliza um desafio para a formação em Psicologia, bem como uma saída para a concretização de ações de saúde integrais orientadas para a inserção e problematização da saúde mental como componente intrinsecamente ligado à saúde geral da comunidade (Scarcelli, \& Junqueira, 2011). A criação de espaços de discussões permite reflexões e construções para o aprimoramento do serviço prestado. Assim, se torna fundamental para a prática o diálogo entre os profissionais, com vistas a romper o isolamento e a hierarquização no campo de trabalho.

Como profissional presente nas Unidades Básicas de Saúde (UBS), o psicólogo deve estar atento à avaliação de seus serviços e práticas. É importante se ater às orientações e críticas ao seu trabalho surgidas não somente no contexto da Psicologia, mas também fora do seu campo (Brasil, 2004; Lima, 2005). Com isso, poder-se-á colher informações relevantes para fundamentar decisões sobre formas mais adequadas de atuação.

Os profissionais de saúde consideram o psicólogo importante para a composição da equipe, no entanto, sua atuação não é claramente definida. Em geral lhe é atribuída a função de oferecer apoio emocional ao paciente, à família e à equipe. Dentre os profissionais da equipe multidisciplinar, o psicólogo é o profissional cujo papel é o mais "obscuro", os demais profissionais não verbalizam como esse apoio deveria acontecer e qual seu significado (Saar, \& Trevizan, 2007). Os psicólogos, por sua vez, se queixam da dificuldade em articular ações de saúde mental no âmbito da atenção básica, seja dentro da própria unidade ou com outros serviços. São dificuldades relativas à ausência de uma rede, de fato interligada, e ao relacionamento entre profissionais da equipe e usuários de saúde mental. $\mathrm{O}$ atendimento oferecido por esses profissionais é, por vezes, de má qualidade, devido à falta de conhecimentos ou mesmo à dificul- 
dade na aceitação do paciente (Conselho Federal de Psicologia, Centro de Referência Técnica em Psicologia e Políticas Públicas, 2010).

As limitações na atuação podem decorrem da formação acadêmica, que ainda não os capacita a transcender o foco de atuação individual para uma abordagem voltada à saúde coletiva. A inserção incipiente do profissional psicólogo na equipe de saúde básica dificulta sua capacitação continuada e a legitimidade de seu trabalho nesse contexto (Fermino, Patrício, Krawulski, \& Sisson, 2009). De acordo com Oliveira, Silva e Yamamoto (2007), a Psicologia não se insere de forma enfática no contexto da Estratégia de Saúde da Família, sendo alvo de críticas acerca de sua atuação no sistema público de saúde. Tal apreensão talvez explique a percepção dos psicólogos de seu trabalho é importante na saúde, mas afirmam não ter pleno conhecimento de suas atribuições. E nem competência suficiente para atender a demanda dos serviços de saúde coletiva (Fermino et al., 2009).

O desafio para o estabelecimento de práticas mais efetivas no campo da saúde envolve a compreensão do conceito de subjetividade. Para compreender um tecido social completo é também necessário incluir na análise o tecido psicológico, do qual o elemento central é o indivíduo. Desse modo, se faz necessário introduzir na concepção das políticas públicas de saúde às contribuições de campos de conhecimento que trabalhem com o conceito de subjetividade, como, por exemplo, a Psicologia (Brasil, 2004). Mesmo após 40 anos de sua inserção no setor de saúde pública, cabe dar continuidade as pesquisas sobre a atuação do psicólogo. A aproximação com a prática demonstra conflitos na delimitação do papel dos psicólogos na atenção básica (Böing, \& Crepaldi, 2010).

\section{Apoio matricial e atenção básica}

A atenção básica à saúde no Brasil é orientada segundo os princípios da Estratégia de Saúde da Família. Implementada em 1994, essa proposta visa a reorganização da atenção básica com o intuito de resgatar os princípios de equidade, universalidade e integralidade do SUS, garantindo o acesso da população a ações em saúde no seu território.

A instituição e ampliação da Estratégia de Saúde da Família gerou transformações nas práticas de saúde mental (Ferreira Neto, 2010). A reorganização da atenção básica apresenta importantes conflu- ências com a proposta da Reforma Psiquiátrica. Os princípios de territorialização e acompanhamento longitudinal devem favorecer a construção de ações inovadoras de promoção, prevenção e reabilitação em saúde mental (Souza et al., 2012).

A Reforma Psiquiátrica apontou a necessidade de se construir um modelo humanizado de atenção em saúde mental. O tratamento deixou de ser centrado na hospitalização para buscar a melhoria da qualidade de vida do usuário, com ênfase em práticas integrais e de promoção à saúde, buscando a autonomia e a cidadania dos pacientes (Simões, Fernandes, \& Aiello-Vaisberg, 2013).

O modelo teórico-prático utilizado atualmente para articular saúde mental e atenção básica é o do apoio matricial, cujo objetivo é integrar ambas por meio de práticas de cuidados colaborativos. Configura-se como uma proposta que visa superar a lógica tradicional médico-centrada no sistema de saúde, como encaminhamentos, referência e contrarreferência, protocolos e centros de regulação; os quais tornaram as relações burocráticas, hierárquicas $\mathrm{e}$ pouco resolutivas (Brasil, 2011).

O trabalho, orientado pelo apoio matricial às equipes de atenção básica, se dá por meio de parcerias e trabalho conjunto de corresponsabilização pela clientela de saúde mental. Devendo ser distinto do atendimento realizado por especialista na unidade de atenção básica (Ferreira Neto, 2010). Desse modo, o apoio matricial não implica em atividades tais como: encaminhamento ao especialista, atendimento individual pelo profissional de saúde mental e intervenção psicossocial coletiva realizado apenas pelo profissional de saúde mental. Mas "um suporte técnico especializado que é ofertado a uma equipe interdisciplinar em saúde a fim de ampliar seu campo de atuação e qualificar suas ações" (Figueiredo, \& Campos, 2009).

De um modo geral, o apoio matricial consiste em encontros interdisciplinares entre profissionais que trabalham nos serviços de saúde mental (psicólogo, psiquiatra, terapeuta ocupacional e outros) e profissionais que trabalham nas unidades de atenção básica (médico, enfermeiro, agente comunitário e outros) para que os primeiros auxiliem os segundos, principalmente a respeito da avaliação e do atendimento de casos que precisam de atenção em saúde mental. O objetivo é que possam acolher e acompanhar alguns casos que talvez não necessitem de atenção especia- 
lizada (Silveira, 2012). Assim, a equipe de Saúde da Família (ESF) responsabiliza-se pelos pacientes que necessitam de cuidados em saúde mental e as equipes de saúde mental (ESM), por sua vez, fornecem orientação e capacitação técnica para as ESFs atenderem essa clientela, por meio de reuniões, debates no serviço e discussões de casos. Espera-se com isso que a atuação conjunta entre ESM e ESF propicie o intercâmbio cotidiano de saberes e competências.

Uma das grandes contribuições do apoio matricial seria a de incentivar a corresponsabilização das equipes de saúde da atenção básica pela demanda de saúde mental. No entanto, o modelo da assessoria e supervisão às ESFs ainda mantém a saúde mental como especialidade, e não como um elemento da saúde integral (Böing, \& Crepaldi, 2010).

Com vistas a contribuir para a discussão e fortalecimento de uma atuação profissional crítica e orientada para a concretização dos princípios do Sistema Único de Saúde, este artigo, objetivou investigar a percepção dos profissionais das unidades básicas de saúde sobre a atuação dos psicólogos nesse setor.

\section{Método}

Esta pesquisa se insere entre as pesquisas qualitativas. Na execução das diversas etapas deste trabalho, destaca-se a importância do investigador como instrumento, o foco no processo e a busca de significados (Bogdan, \& Biklen,1994). A coleta de dados ocorreu em duas etapas. Na primeira aplicou-se um questionário (questões abertas e fechadas) ao maior número de sujeitos possível, considerando o universo dos profissionais de saúde atuantes nas Unidades Básicas de Saúde existentes. Obteve-se um total de 54 colaboradores. Após, realizou-se uma entrevista semiestruturada, em uma amostra de 14 profissionais, constituída pelos sujeitos que responderam anteriormente ao questionário. Desse modo obteve-se uma 'amostra da amostra', procedimento respaldado por de Spink (2004) quando a amostra é grande.

Colaboraram, neste estudo, profissionais das categorias preconizadas para as equipes das Unidades Básicas de Saúde, a saber: médicos, enfermeiros, dentistas, assistentes sociais, assistentes administrativos e agentes comunitários de saúde. Como os psicólogos também realizavam atendimentos nas unidades e, com base no objetivo desta pesquisa, eles também foram inseridos como colaboradores.
O objetivo desse procedimento foi resguardar cada uma das equipes existentes na cidade de Uberlândia, pois a escolha dos sujeitos correspondeu, em número de categorias profissionais, a duas equipes típicas de UBSs (14 profissionais, sendo dois de cada categoria profissional acima mencionada), sem serem pertencentes a uma mesma equipe. Desse modo, evitou-se a exposição da identidade das equipes das UBSs. As entrevistas se basearam nas considerações de Alonso (1995), caracterizando-se por serem semiestruturadas e baseadas em um roteiro, contemplando os seguintes temas: formação dos psicólogos, Unidades Básicas de Saúde, equipes multidisciplinares, relação dos psicólogos com outros profissionais e expectativas e avaliação do trabalho do psicólogo.

Os dados coletados foram analisados sob duas perspectivas: as questões fechadas do questionário, passíveis de tabulação, receberam tratamento estatístico descritivo. As questões abertas dos questionários, que tratam de temas ou explicações de respostas às questões fechadas, foram analisadas em conjunto com as entrevistas, permitindo assim a mesma forma de análise. Neste trabalho, devido ao espaço, privilegia-se a análise das entrevistas e das questões abertas respondidas no questionário, as quais foram analisadas conjuntamente.

A análise das entrevistas deu-se após sua escuta e transcrição, momentos em que foram realizados anotações e comentários, já de cunho analíticos, suscitado pelas falas dos colaboradores. Com o material transcrito fez-se uso de uma tabela no Microsoft Word, em três colunas e número de linhas indeterminado. Na primeira coluna foi colada a entrevista bruta, da qual se extraía trechos significativos, colados na segunda coluna e, finalmente, na terceira coluna, na mesma linha, inferências quanto aos sentidos das afirmações e relações com a literatura, sob a tarja de um tema que o fragmento sugeria aos pesquisadores. Realizada essa etapa, juntaram-se os fragmentos das diferentes entrevistas alocados sob o mesmo tema. Tomou-se como critério nesse procedimento a repetição temática e o sentido que veiculavam. Em seguida, promoveu-se um depuramento, eliminando repetições e afirmações deslocadas do tema.

Ao fim, o trabalho analítico resultou em quatro categorias, cujos títulos as definem. Suprimiu-se qualquer identificação dos colaboradores, inclusive as relativas à formação, uma vez que apenas dois de cada categoria responderam a entrevista e poderiam 
ser facilmente identificados. Isso gera, com certeza, alguns prejuízos, mas é um cuidado ético e um incentivo para que novas pesquisas sobre o tema sejam realizadas com profissionais específicos. E, obviamente, maior número de sujeitos para que os participantes sejam diluídos entre eles.

As falas foram editadas, sem qualquer prejuízo de sentido. Observou-se nesse procedimento que as entrevistas convergiam para os seguintes temas: trabalho dos psicólogos nas Unidades Básicas de Saúde, relação dos psicólogos com a equipe, relação de sua formação com o trabalho e avaliação de sua atuação. Em conformidade com a Resolução $\mathrm{n}^{\circ}$ 196/96, vigente na época, esta pesquisa foi submetida ao Comitê de Ética em pesquisa da Universidade Federal de Uberlândia, sob o número de protocolo 050/009, tendo sido devidamente aprovada no dia 15 de maio de 2009.

\section{Resultados e Discussão}

A articulação, na prática, entre saúde mental e atenção básica impõe diversos desafios a serem negociados no cotidiano de trabalho. Dentre eles, a elevada demanda, a prevalência do modelo assistencial psiquiátrico tradicional em detrimento das abordagens substitutivas e o despreparo dos profissionais das ESFs para lidar com problemas de saúde mental (Prates, Garcia, \& Moreno, 2013). Os resultados apontaram que ainda há um longo caminho para a efetiva articulação desses dois grandes campos. Sendo que o trabalho do psicólogo é um fator de análise e avaliação para o modo como tem sido implementada essa articulação.

De forma geral, os participantes consideram o psicólogo um importante membro para a realização de um trabalho em equipe (30,4\%), apesar de realizarem poucas atividades em conjunto $(26,6 \%)$. A parceria, quando acontece, se dá em maior número, através de solicitações do próprio psicólogo (29\%) se comparada às solicitações dos demais profissionais $(25,8 \%)$. Referem que não há um trabalho em equipe, e sim uma lógica de encaminhamento dentro da própria Unidade. A falta de atuação conjunta não se dá por indisposição dos profissionais, mas pela dificuldade de sua operacionalização. Há a percepção e a demanda por atividades em conjunto, porém não há a sua realização.

Os profissionais ficam envolvidas nas atividades de seu núcleo específico de trabalho, restando pouco tempo para a atuação em equipe. A grande quantidade de atendimentos das UBSs é um agravante nesse contexto, empurrando os profissionais para a solução imediata da demanda, dificultando um planejamento e execução de atividades multiprofissionais. Observa-se, com isso, que o trabalho do psicólogo acaba ficando circunscrito às atividades de sua área de excelência, a saúde mental. Apesar de outras atividades, como grupos $(20,2 \%)$ e visitas domiciliares $(11,4 \%)$, serem citadas pelos profissionais como oportunidades de contato com o trabalho do psicólogo.

\section{Categoria 1 - 0 trabalho do psicólogo no setor de saúde}

Os profissionais das unidades básicas de saúde compreendem que a realização de grupos, atendimentos de demanda livre, retornos de atendimentos, orientações e encaminhamentos são as atividades principais realizadas pelos psicólogos, sendo elas circunscritas à saúde mental. A demanda que não se circunscreve nessa característica, geralmente, não é contemplada pelas práticas do psicólogo. Por exemplo, o psicólogo realiza visitas e grupos para pacientes inseridos no Programa de Saúde Mental do município, sendo rara a sua participação em grupos de hipertensos e diabéticos, pertencentes a outros Programas da unidade.

Desse modo, os profissionais afirmam que sobra pouco tempo para a atuação do psicólogo junto às outras demandas da unidade de saúde, tendo em vista que a demanda de saúde mental é volumosa frente ao número de psicólogos (120 para uma população total de 604.013 mil habitantes). Com isso o trabalho em equipe fica comprometido: "os programas de saúde mental têm sido muito importantes, mas não sobra tempo para ela [psicóloga] participar com a equipe".

Apesar de estar inserido numa unidade básica de saúde, o psicólogo fica encarregado, quase que exclusivamente, das demandas de saúde mental. Com isso seu trabalho fica isolado do restante da unidade, que cuida das outras demandas dos pacientes. Pode-se afirmar que a troca de experiências e corresponsabilização do cuidado, proposta pelo apoio matricial, não se efetiva na prática.

O trabalho do psicólogo, inserido na atenção básica, sofreu transformações. Destaca-se sua a falta de conhecimento acerca do papel do psicólogo nas equipes e o aumento da demanda reprimida para 
a Psicologia (Oliveira et al., 2007). A falta de conhecimento do restante dos profissionais sobre o apoio matricial, ocasiona dificuldade de comunicação a respeito do papel do psicólogo na unidade. Algo que pode ser exemplificado com uma das solicitações dos profissionais, que é a realização de terapia de grupo para os profissionais da unidade básica de saúde. A solicitação de acompanhamento institucional da equipe é legítima e aponta para a necessidade dos profissionais de gestão do SUS articularem formas de cuidado das equipes de saúde. Porém, ela não está descrita como uma das funções do psicólogo na atenção básica. E, devido ao seu vínculo como funcionário da unidade, não poderia exercer, de bom grado, a função de supervisor institucional.

A realização da escuta foi avaliada pelos profissionais como a especificidade e a contribuição principal dos psicólogos para o serviço, "[os pacientes] querem que alguém os ouça e o psicólogo tem essa especificação". Avaliada positivamente, a escuta auxilia o restante da equipe no trabalho junto ao paciente. Trechos como: "ajudam a todos os profissionais" e "Muitos pacientes usam medicação quando na verdade eles precisavam de espaço para fazer terapia" apontam que a forma de lidar com o sofrimento dos pacientes é considerada uma especialidade dos psicólogos, quase uma espécie de dom: “(...) é uma habilidade única ver o que realmente está causando sofrimento". As percepções dos profissionais apontam a necessidade dos psicólogos orientarem sua prática, constantemente, para uma qualidade apurada da escuta.

Para alguns profissionais, a prática da clínica tradicional ainda é uma forma de reconhecer o psicólogo (Archanjo, \& Schraiber, 2012). Os relatos apontaram que a definição da especificidade do trabalho psicológico são atividades que envolvem escuta e terapia. Práticas que envolvem outros profissionais, como grupo educativo e atividades comunitárias, por vezes, não foram reconhecidas como práticas psi legitimadas. Isto demonstra que ainda há um acompanhamento fragmentado do paciente: o psicólogo cuida da mente e o restante da equipe cuida do corpo, inviabilizando a proposta do Sistema Único de Saúde para um acompanhamento integral visando a promoção e a prevenção, além das práticas puramente curativas.

A aplicação da clínica tradicional à saúde pública, por parte dos psicólogos é percebida como negativa pelos profissionais "às vezes eles não se envolvem com o trabalho de campo mesmo" e "esperamos que eles façam um trabalho não só voltado para dentro do consultório deles (...) mas que inclua os outros profissionais. Desse modo, os profissionais de saúde entendem que os psicólogos não conhecem as políticas públicas, optando por uma prática clínica tradicional, seja por uma escolha pessoal ou por disporem somente dela como ferramenta: "o trabalho do psicólogo dentro da atenção básica fica completamente prejudicado".

Nas unidades básicas de saúde observadas, a equipe de saúde mental (ESM) era composta por psicólogo e o psiquiatra. No dia a dia de trabalho, o psicólogo estava presente na unidade de saúde quatro vezes na semana. Já o psiquiatra tinha contato com a equipe, através do clínico geral, que atendia os pacientes do Programa de Saúde mental, uma vez no mês.

De um modo geral, o psicólogo faz atendimentos individuais e em grupos com os pacientes, sem a presença de outros profissionais da unidade. O trabalho do psiquiatra se dá, essencialmente, através de uma atividade nomeada tutoria, em que se reúnem o psiquiatra, o clínico geral e o paciente, para a discussão sobre a medicação psicotrópica. Por vezes, o psicólogo não participa dessa atividade, por julgar não pertencer à sua área de saber e, assim, avalia que tem pouco a contribuir. Os relatos demonstram também uma sensação de desconforto em participar de uma atividade em que é discutida questões psicotrópicas. As práticas observadas vão claramente de encontro com as propostas do apoio matricial, uma vez que são atividades realizadas individualmente pelos profissionais da equipe de saúde mental, sem o envolvimento dos demais profissionais da unidade. Com isso, compromete-se a proposta de cuidados corresponsabilizados prevista nas atividades de matriciamento.

Os cuidados dos pacientes com sofrimento mental ficam a cargo, quase que exclusivamente, do psicólogo, pois ele está um maior período na unidade. Os demais profissionais, envolvidos em outras atividades, não participam do acompanhamento dos pacientes da saúde mental, comprometendo a troca de conhecimentos entre ESM e ESF, além o fato de não terem instrumentos para intervir numa situação de crise psíquica, quando necessário.

$\mathrm{Na}$ avaliação dos profissionais, há uma boa relação entre a comunidade e os psicólogos, "aqui a adesão é muito boa (...) não tem aquele preconceito de antigamente", "a comunidade necessita", 
"a procura é grande, eles criam vínculo e se apegam". Os psicólogos, por sua vez, afirmam que a comunidade busca muito o seu trabalho, por demanda espontânea ou encaminhamento dos médicos. Porém, vale ressaltar, que a busca se dá, em sua maioria, por práticas tradicionais de atendimento, como a terapia individual.

Para atender à população, os psicólogos priorizam os grupos, devido à grande demanda ou por acreditarem na efetividade dessa modalidade. Eles relataram que alguns pacientes se sentem desassistidos quando são atendidos na modalidade de grupo, queixando-se na coordenação da unidade, ou mesmo na mídia, da falta de psicólogos. Essa queixa reflete a percepção da Psicologia como uma atividade exclusivamente de tratamento individual.

Em seu aspecto conceitual, o apoio matricial possibilita o processo de reabilitação psicossocial da pessoa em sofrimento mental, por suas práticas se darem na comunidade e por suas modalidades de cuidado acontecerem no âmbito da atenção básica. Entretanto, a prática aponta que a assistência prestada ainda traz em seu bojo conceitos e práticas que remetem à psiquiatria e à Psicologia tradicional (Machado, \& Pereira, 2013).

\section{Categoria 2 - Trabalho em equipe}

O trabalho em equipe, conforme apontado na categoria 1, é ainda pouco realizado na atenção básica. Quando acontece se dá por meio de visitas domiciliares e de atendimento a pacientes em crise: "nós discutimos sobre pacientes em crise (...) nessa hora a ponte é bem feita. Já fiz visita domiciliar com psicólogo e eles ajudaram bastante". O relato aponta que é possível trabalhar em equipe. No entanto, elas têm acontecido em situações de crise, com isso, observa-se que há que se construir, em termos de práticas de trabalho, condições para que o trabalho em equipe seja uma rotina.

A atuação conjunta acontece quando alguma demanda extrapola a área de conhecimento do profissional, então ele solicita o psicólogo ou vice-versa. Essa parceria poderia ampliar as possibilidades de atendimento aos pacientes, que não aquelas somente médico-centradas, "ficar só trocando remédio não resolve". No entanto, ela ainda não é uma prática comum nas atividades das unidades, sendo utilizada geralmente em momentos críticos.
Através do trabalho em equipe, os profissionais acreditam que os serviços na unidade seriam otimizados, tanto no atendimento ao paciente, como nas relações que se estabelece entre os membros: “(o psicólogo) ajuda bastante na relação com o grupo. Nas relações extra-atendimento, na demanda normal (...) a lidar com o ser humano, a facilitar o atendimento". Na percepção dos psicólogos o trabalho em equipe é premissa para atuação na saúde. "Não tem outro jeito de trabalhar em Saúde pública, se não for em equipe (...) nunca sou eu sozinha (...) o paciente não é da psicóloga, é da equipe". Porém, os demais relatos apontam ser ainda um grande desafio.

\section{Categoria 3 - Formação profissional dos psicólogos}

Houve destaque para o fato de que nenhum profissional da rede de saúde recebe formação acadêmica apropriada para o trabalho em saúde pública: "Nem psicólogo, nem médico. Ninguém é direcionada para essa área pública”. Aponta uma formação acadêmica pouco vinculada à futura realidade de trabalho de grande parte dos alunos, uma vez que o setor público é o grande empregador, principalmente dos recém-formados. Por mais adequada que tenha sido a formação, há falhas, devido à complexidade da clientela atendida e às relações em equipe. Os psicólogos afirmam que a Psicologia não os capacitou para a prática em saúde pública e que essa aprendizagem se deu na prática: "não saímos da faculdade preparados (...) a minha atuação foi se construindo mesmo na prática”.

\section{Categoria 4 - Avaliação da atuação do psicólogo}

De modo geral, a avaliação do trabalho do psicólogo foi positiva, considerando-se as limitações já apontadas. Partindo de diversos pontos, os profissionais enumeraram situações as quais refletem sua avaliação. A importância do trabalho do psicólogo foi compreendida como relevante devido à sua capacidade de oferecer suporte: "é um suporte que nós temos; ajuda a resolver todo o tipo de problemas". Há uma atribuição muito ampla às contribuições do psicólogo, chegando ao ponto de considerarmo-las um tanto "milagrosas". Outro aspecto avaliativo foi a percepção da possibilidade de um diálogo diferen- 
ciado, orientado para a melhoria das condições de vida dos pacientes: "eles têm uma conversa mais específica, orientando o paciente, dando uma nova visão".

De um modo geral, os profissionais de saúde queixam-se da falta de psicólogo. O baixo número de atendimentos, comparados à demanda, é atribuído, não à incapacidade dos profissionais, mas ao aumento da população e às dificuldades de operacionalização da oferta de saúde mental à população: “(...) a demanda é muito grande. Deveria ter mais um profissional. A área de abrangência é muito grande e deixa o trabalho deficitário".

Embora os profissionais da atenção básica possam fornecer a maior parte dos cuidados, os profissionais de saúde mental (psiquiatras, psicólogos, enfermeiros e especialistas em saúde psicossocial) são necessários para gerenciar o acompanhamento dos pacientes. Sem esses profissionais de saúde mental, os países periféricos não têm recursos humanos suficientes para atender às solicitações de tratamento em saúde mental de suas populações (Bruckner et al., 2011).

A organização do atendimento em saúde mental para atenção básica, através do apoio matricial, aponta como a gestão dos recursos humanos altera as premissas da própria política de apoio matricial. Para atender à população, de modo mais amplo, quantitativamente, os psicólogos foram inseridos na rotina das unidades. Houve um enveredamento para a realização de atividades da clínica tradicional, por serem elas de maior domínio técnico dos psicólogos, diante da enorme demanda da comunidade. Com isso, o ato de matriciar ficou negligenciado. Desse modo, os psicólogos são alocados, mas não necessariamente estão disponíveis para as unidades. Atendem à demanda de saúde mental, por pertencerem à equipe de apoio matricial, mas não estão disponíveis para atividades voltadas às demais demandas em saúde. Observa-se, devido a isso, uma apreensão e aplicação parcial da proposta de cuidados compartilhados do apoio matricial.

Outra queixa relevante foi o fato do psicólogo não estar inserido na equipe mínima do Programa de Saúde da Família: “(...) se tivesse o profissional junto com a equipe de PSF eu acho que o trabalho seria bom”. Os profissionais apoiam a inserção do psicólogo como profissional da equipe mínima do Programa de Saúde da Família. Os psicólogos relatam atuar dentro das possibilidades, dado o modo como está organizada a atenção básica: "A gente está atuando até demais". Os relatos apontam uma expectativa de múltipla atuação para os psicólogos: atendimentos de saúde mental, psicoterapia institucional, atividades comunitárias e atendimento à saúde integral. Porém, o psicólogo não possui recursos de tempo e humano para exercer essas múltiplas funções.

\section{Conclusão}

O primeiro cuidado importante quando se conclui um trabalho como esse é apontar seus limites. Um deles é que o conjunto de colaboradores tece respostas ao trabalho de um grupo restrito de psicólogos da saúde pública em Uberlândia, em uma população total que é no mínimo 12 vezes maior. O segundo, é a tentação sempre presente de conclusões generalizadoras. Diante de nossos objetivos, entendemos que a descrição das categorias permite aos leitores, conclusões parciais, de resto, impossíveis de serem explanadas exaustivamente nos limites desse trabalho.

Dentro dos limites apontados, os resultados corroboram a literatura, a qual aponta as dificuldades encontradas pelos psicólogos em sua atuação na saúde pública. Uma delas é da ordem das políticas de saúde, estabelecidas pelas linhas programáticas que de certa forma aprisionam os psicólogos às demandas da saúde mental e, dentro dessa, privilegia um tipo de atuação e clientela.

Decorrente disso, elevados ao lugar de herdeiros das respostas científicas e sanitárias à saúde mental, os psicólogos encontram uma comunidade com uma expectativa de respostas tradicionais, especialmente as relativas à saúde mental. Em geral, a solução dos problemas e a terapia são os recursos e atos mais solicitados, na medida em que se atribui tais funções aos psicólogos. Desse modo, confessa-se a própria falta de recursos, das unidades de saúde, para atendê-las, o que de certa forma, nega a afirmação, tantas vezes repetidas, de que os pacientes não são de um único profissional, mas da equipe. Inúmeras vezes, na prática, o paciente é somente do psicólogo, a despeito de sua vontade. E essa expectativa surge acompanhada de uma cobrança implícita das equipes: de que as demandas sejam acolhidas e os problemas resolvidos, o que implicitamente, se configura como um alívio para as questões de múltiplas causas. 
Nesse sentido, a representação mais precisa que se tem do psicólogo assemelha-se à do coringa de baralho, que serve para tudo, porém, não tem um lugar específico na sequência das cartas. Em outras palavras, parece que o papel dos psicólogos nas unidades básicas de saúde é institucionalmente mal definido, ele está presente na unidade, mas não faz parte da equipe mínima, aspecto atribuído, em grande parte às políticas de saúde, que não favorecem sua inclusão.

Cabe aludir em relação a isso dois aspectos: o primeiro, o psicólogo ainda carece de uma identidade clara quando atua na saúde pública, recebendo da equipe demandas relativas ao novo modelo de atenção básica, que não visa procedimentos como a psicoterapia. Com relação às demandas para a atuação conjunta, observa-se que ao atendê-las, deixa-se de acolher as constantes solicitações de resolutividade, que chegam principalmente por meio dos auxiliares administrativos e agentes de saúde.

O segundo aspecto, é que os próprios psicólogos se ressentem da formação recebida na academia, uma vez que um de seus melhores recursos, a terapia, mostra-se inadequada para o cotidiano da saúde. A solução, como aponta uma dessas profissionais, é capacitar-se na prática, a despeito da formação - e atuação - tradicional estar no imaginário de inúmeros profissionais. Há que se destacar que dois elementos, inerentes à prática tradicional de Psicologia clínica, mostram-se eficientes aos olhos das equipes: a capacidade de escutar a clientela e o estabelecimento de vínculo que viabiliza a adesão.

A escuta tem um papel fundamental na atuação do psicólogo. Compreendendo-a em seu sentido ampliado, nas instituições de saúde ela torna possível problematizar a percepção da equipe e dos próprios usuários a respeito dos problemas suscitados. Pelo seu papel de escuta, o psicólogo pode atuar como um intermediador entre usuários e sistema de saúde, "limpando os canais de comunicação" (Pietroluongo, \& Resende, 2007).

Outro papel atribuído ao psicólogo dentro das equipes é o acolhimento da própria equipe, propiciando a construção de vínculos, que, cremos, se dá no acolhimento da própria equipe. Este acolhimento, segundo as autoras citadas acima, é tido como essencial em momentos de não saber da equipe. A escuta psicológica pode auxiliar no modo como lidar com este não saber, bem como encontrar novas saídas.
Delineia-se com um isso uma visão da importância e do papel que se dá a esse profissional: o de mediador, entre as políticas públicas, e entre a equipe e a comunidade assistida.

Por fim, cabe assinalar que há uma expectativa por uma maior presença física dos psicólogos nas unidades básicas de saúde, tanto no sentido de acolher a demanda "por resolutividade", como no sentido de um acolhimento e uma integração maior com a equipe. De modo geral a equipe entende, com destaque para os enfermeiros e assistentes sociais, que os psicólogos são profissionais cindidos: por um lado são colocados como um dos agentes principais de um importante programa de saúde pública, o do apoio matricial. E, por outro lado, são considerados peças importantes e necessárias em programas que não tem essa especificidade, como o Programa de Saúde da Família.

Enfim, a despeito das expectativas das equipes das unidades básicas de saúde, há a expectativa institucional de que os psicólogos se desdobrem no atendimento dessas duas questões: atenuar os problemas de saúde mental e envolver-se nas demandas de saúde geral da atenção básica. Vozes como a dos agentes comunitários podem estar corretas quando afirmam que são poucos profissionais para a grande demanda. No mínimo, para as duas demandas, que requerem profissionais com perfis distintos.

A atuação do psicólogo como profissional de ligação de saúde mental e atenção básica tem promovido mudanças nas Unidades Básicas de Saúde. Porém, é necessário uma profunda reflexão e maior envolvimento dos profissionais, incluindo psicólogos, e dos gestores para a viabilização de mudanças estruturais para a construção de uma efetiva prática integrada. E, se necessário, inclusive refletir sobre a política de saúde mental para a atenção básica, principalmente quando o cotidiano lhes conte diariamente, das dificuldades de sua execução.

Os resultados apontaram questões que podem fomentar o debate sobre o tópico e a realização de outras pesquisas, essencialmente sobre a aplicabilidade das políticas de saúde à realidade das unidades de saúde, que, por diversos fatores, mostram não conseguirem executá-las. É preciso refletir sobre a prática cotidiana, de modo que sirva de termômetro para a efetividade e eficácia das políticas de saúde. 


\section{Referências}

Alonso, L. E. (1995). Sujeito y discurso: el lugar de la entrevista abierta em las práticas de la sociología cualitativa. In J. M. Delgado, \& J. Gutierrez, Metodos y tecnicas cualitativas de Investigação em ciencias sociales (pp. 225-240). Madrid: Síntesis.

Andrade, J. F. S. M., \& Simon, C. P. (2009). Psicologia na atenção primária à saúde: reflexões e implicações práticas. Paidéia (Ribeirão Preto), 19(43), 167-175. doi:10.1590/S0103-863X2009000200005

Archanjo, A. M., \& Schraiber, L. B. (2012). A atuação dos psicólogos em unidades básicas de saúde na cidade de São Paulo. Saúde e Sociedade, 21(2), 351-363. doi:10.1590/S0104-12902012000200009

Azevedo, L. A., Tatmatsu, D. I. B., \& Ribeiro, P. H. R. (2011). Formação em Psicologia e a apropriação do enfoque da atenção primária à saúde em Fortaleza, Ceará. Trabalho, Educação e Saúde,9(2), 241-264. doi:10.1590/S1981-77462011000200005

Bernardes, J. (2006). Formação generalista em Psicologia e Sistema Único de Saúde. In Conselho Federal de Psicologia. (Ed.), Fórum Nacional de Psicologia e Saúde Pública: contribuições técnicas e políticas para avançar o SUS (Vol 1). Brasília, DF: O autor.

Bogdan, R. C., \& Biklen, S. K. (1994). Investigação qualitativa em educação: uma introdução à teoria e ao método. Porto: Porto Editora.

Böing, E., \& Crepaldi, M. A. (2010). O psicólogo na atenção básica: uma incursão pelas políticas públicas de saúdebrasileiras. Psicologia:CiênciaeProfissão,30(3), 634-649. doi:10.1590/S1414-98932010000300014

Brasil, A. M. R. C. (2004). Considerações sobre o trabalho do psicólogo em saúde pública. Integração, 10(37), 181-186. Recuperado de ftp://ftp.usjt.br/ pub/revint/181_37.pdf

Brasil. (2011). Ministério da Saúde. Saúde mental no SUS: as novas fronteiras da reforma psiquiátrica: relatório de gestão 20072010. Brasília, DF: o autor.

Bruckner, T. A., Scheffler, R. M., Shen, G., Yoon, J., Chisholm, D., Morris, J.,... Shekhar, S. (2011). The mental health workforce gap in low- and middle-income countries: a needs-based approach. Bulletin of the World Health Organization, 89(3), 184-194. Recuperado de http://www.who.int/bulletin/volumes/89/3/10-082784/en/

Conselho Federal de Psicologia, Centro de Referência Técnica em Psicologia e Políticas Públicas (Orgs.).
(2010). Práticas profissionais de psicólogos e psicólogas na atenção básica à saúde. Brasília, DF: Conselho Federal de Psicologia.

Dimenstein, M. D. B. (1998). O psicólogo nas Unidades Básicas de Saúde: desafios para a formação e atuação profissionais. Estudos de Psicologia (Natal), 3(1), 53-81. doi:10.1590/S1413-294X1998000100004 Fermino, J. M., Patrício, Z. M., Krawulski, E., \& Sisson, M. C. (2009). Atuação de psicólogos no Programa de Saúde da Família: o cotidiano de trabalho oportunizando repensar a formação e as práticas profissionais. Aletheia, (30), 113-128. Recuperado de http:// pepsic.bvsalud.org/scielo.php?script=sci_arttext\&pid=S1413-03942009000200010

Ferreira Neto, J. L. (2010). A atuação do Psicólogo no SUS: análise de alguns impasses. Psicologia: Ciência e Profissão, 30(2), 390-403. doi:10.1590/S1414-98932010000200013

Figueiredo, M.D., \& Campos, R. O. (2009). Saúde mental na atenção básica à saúde de Campinas, SP: uma rede ou um emaranhado? Ciência \& Saúde Coletiva, 14(1), 129-138. doi:10.1590/S1413-81232009000100018

Lima, M. (2005). Atuação psicológica coletiva: uma trajetória profissional em Unidade Básica de Saúde. Psicologia em Estudo (Maringá), 10(3), 431-440. doi:10.1590/S1413-73722005000300011

Machado, M. P., \& Pereira, M. A. O. (2013). Percepção da doença mental por profissionais de saúde: possibilidades de ampliação do cuidado. Estudos $e$ Pesquisas em Psicologia, 13(1), 125-138. Recuperado de http://www.revispsi.uerj.br/v13n1/artigos/pdf/ v13nla08.pdf

Nepomucemo, L. B., \& Brandão, I. R. (2011) Psicólogos na estratégia saúde da família: caminhos percorridos e desafios a superar. Psicologia: Ciência e Profissão, 31(4), 762-777. doi:10.1590/S1414-98932011000400008

Oliveira, I. F., Silva, F. L., \& Yamamoto, O. H. (2007). A psicologia no Programa de Saúde da Família (PSF) em Natal: espaço a ser conquistado ou um limite da prática psicológica? Aletheia, (25), 5-19. Recuperado de http://pepsic.bvsalud.org/scielo.php?script=sci_arttext\&pid=S1413-03942007000100002

Pietroluongo, A. P. C., \& Resende, T. I. M. (2007). Visita domiciliar em saúde mental: o papel do psicólogo em questão. Psicologia: Ciência e Profissão, 27(1), 22-31. doi:10.1590/S1414-98932007000100003 
Portes, J. R. M., \& Máximo, C. E. (2010/2). Formação do psicólogo para atuar no SUS: possíveis encontros e desencontros entre as Diretrizes Curriculares Nacionais e as Matrizes Curriculares de um curso de Psicologia. Barbarói, (33), 153-177. doi:10.17058/barbaroi.v0i0.1538

Prates, M. M. L., Garcia, V.G., \& Moreno, D. M. F. C. (2013). Equipe de apoio e a construção coletiva do trabalho em Saúde Mental junto à Estratégia de Saúde da Família: espaço de discussão e de cuidado. Saúde e Sociedade, 22(2), 642-652. doi:10.1590/S0104-12902013000200031

Ronzani, T. M., \& Rodrigues, M. C. (2006). O psicólogo na atenção primária à saúde: contribuições, desafios e redirecionamentos. Psicologia: Ciência e Profissão, 26(1), 132-143. doi:10.1590/S1414-98932006000100012

Saar, S. R.C., \&Trevizan, M.A. (2007). Papéis profissionais de uma equipe de saúde: visão de seus integrantes. $R e$ vistaLatino-americana deEnfermagem, 15(1), 106-112. doi:10.1590/S0104-11692007000100016

Scarcelli, I. R., \& Junqueira, V. (2011). O SUS como desafio para a formação em Psicologia. Psicologia: Ciência e Profissão, 31(2), 340-357. doi:10.1590/S1414-98932011000200011

Silveira, E. R. (2012). Práticas que integram a saúde mental à saúde pública: o apoio matricial e a interconsulta. Ciência \& Saúde Coletiva, 17(9), 2377-2386. doi:10.1590/S1413-81232012000900018

Simões, C. H. D., Fernandes, R. A., \& Aiello-Vaisberg, T. M. J. (2013). O profissional de saúde mental na reforma psiquiátrica. Estudos de Psicologia (Campinas), 30(2), 275-282. doi:10.1590/S0103-166X2013000200014

Souza, L. G. S., Menandro, M. C. S., Couto, L. L. M. Schimith, P. B., \& Lima, R. P. (2012). Saúde mental na estratégia saúde da família: revisão da literatu- ra brasileira. Saúde e Sociedade, 21(4), 1022-1034. doi:10.1590/S0104-12902012000400019

Spink, M. J. (2004) A formação do psicólogo para a atuação em instituições de saúde. Em M. J. Spink. (Org) Psicologia social e saúde: práticas, saberes $e$ sentidos (2a ed.). Petrópolis, RJ: Vozes.

\section{Francielle Xavier Dias}

Mestre pelo Programa de Psicologia da Faculdade de Filosofia, Ciências e Letras de Ribeirão Preto, Universidade de São Paulo. Especialista em Gestão Pública em Saúde pela Universidade Federal de Uberlândia. Graduação em Psicologia pela Universidade Federal de Uberlândia. Uberlândia - MG. Brasil.

E-mail: franciellexdias@usp.br

Luiz Carlos Avelino da Silva, Mestre em Psicologia Clínica pela Universidade de Brasília e Doutor em Psicologia da Educação e Desenvolvimento Humano, pelo Instituto de Psicologia da Universidade de São Paulo. Professor Associado do Instituto de Psicologia, Universidade Federal de Uberlândia. Uberlândia - MG. Brasil.

E-mail: luizavelino@yahoo.com.br

Endereço para envio de correspondência:

Rua Triunfo, 1298, apto 32, Jardim Botânico. CEP: 14021-612. Ribeirão Preto - SP. Brasil.

Recebido 30/07/2014

Aprovado 21/07/2016

Received 07/30/2014

Approved 07/21/2016

Recibido 30/07/2014

Aceptado 21/07/2016

Como citar: Dias, F. X., \& Silva, L. C. A.. (2016). Percepções dos profissionais sobre a atuação dos psicólogos nas unidades básicas de saúde. Psicologia: Ciência e Profissão, 36(3): 534-545. doi: 10.1590/1982-3703001102014

How to cite: Dias, F. X., \& Silva, L. C. A.. (2016). Perceptions of other professionals about the work of psychologists in health basic units. Psicologia: Ciência e Profissão, 36(3): 534-545. doi: 10.1590/1982-3703001102014

Cómo citar: Dias, F. X., \& Silva, L. C. A.. (2016). Percepciones de otros profesionales sobre la actuación de psicólogos en unidades de atención primaria de salud. Psicologia: Ciência e Profissão, 36(3): 534-545. doi: 10.1590/1982-3703001102014 